\title{
Hemorrhagic presentations of cerebellar pilocytic astrocytomas in children resulting in death: report of 2 cases
}

\author{
Mitchell P. Wilson, MD, ${ }^{1}$ Edward S. Johnson, MD, FRCPC, ${ }^{2}$ Cynthia Hawkins, MD, PhD, FRCPC, ${ }^{3}$ \\ Kerry Atkins, BSc, ${ }^{4}$ Wael Alshaya, MD, ${ }^{5,6}$ and Jeffrey A. Pugh, MD, MSc, FRCSC ${ }^{5}$ \\ Departments of ${ }^{1}$ Radiology and Diagnostic Imaging, ${ }^{2}$ Laboratory Medicine and Pathology, ${ }^{4}$ Faculty of Medicine and Dentistry, \\ and ${ }^{5}$ Division of Neurosurgery, Departments of Surgery and Clinical Neurosciences, University of Alberta, Edmonton, Alberta; \\ ${ }^{3}$ Division of Pathology and Labatt Brain Tumor Research Centre, Hospital for Sick Children, University of Toronto, Ontario, \\ Canada; and ${ }^{6}$ King Abdulaziz Medical City, Ministry of National Guard-Health Affairs, Riyadh, Saudi Arabia
}

Acute hemorrhagic presentation in pilocytic astrocytomas (PAs) has become increasingly recognized. This type of presentation poses a clinically emergent situation in those hemorrhages arising in PAs of the cerebellum, the most frequent site, because of the limited capacity of the posterior fossa to compensate for mass effect, predisposing to rapid neurological deterioration. As examples, we describe two cases of fatal hemorrhagic cerebellar PAs: one of a child with a slowly growing stereotypical WHO Grade I PA with a 1-year period of symptomatology that preceded a rapid clinical deterioration, and another of an asymptomatic child having a PA variant, presenting with progressive obtundation following a presumed Valsalva event. These two scenarios parallel previous reports in the literature of either a setting of progressive expression of cerebellar dysfunction and transient episodes of raised intracranial pressure (ICP), or abrupt onset of features of increased ICP in a previously well child. The literature is further reviewed for a current understanding of the factors that predispose, initiate and propagate bleeding, with specific reference to the role of vascular endothelial growth factor and other angiogenic agents in the genesis and stability of the vasculature in PAs. In this context, we propose that obliterative vascular mural hyalinization with associated altered flow dynamics and microaneurysm formation was the pathogenesis of the hemorrhage in our first case. In the second case, large tumor size, increased growth rate, looseness of the background myxoid matrix, and thinness of the tumor blood vessels with calcospherite deposition predisposed to vascular leakage and bleeding concurrent with sudden increases in intravascular hydrostatic pressure. In that cerebellar PAs are common, this report underscores the importance of considering in the differential diagnosis the possibility of a spontaneous hemorrhage in a posterior fossa PA in a child presenting with a sudden neurological ictus and raised ICP.

http://thejns.org/doi/abs/10.3171/2015.10.PEDS1580

KEY WORDS pilocytic astrocytoma; low-grade astrocytoma; juvenile pilocytic astrocytoma; hemorrhage; pediatric; VEGF; BRAF; vascular disorders

$\mathrm{S}$ PONTANEOUS hemorrhage in pilocytic astrocytomas (PAs) has recently become recognized in the literature as being more common than previously reported $^{11,19,24}$ and is of critical clinical importance in those tumors arising in the cerebellum. One reason is that this site accounts for an estimated $40 \%$ of all PAs occurring in the brains of children, ${ }^{3,6}$ in whom PA comprises $15 \%$ of primary CNS neoplasms. ${ }^{16}$ Another reason is the lim- ited compensatory capacity in the posterior fossa to adjust to sudden increases in mass effect, predisposing to rapid clinical deterioration. In this context, we report 2 illustrative fatal cases of cerebellar PA with spontaneous hemorrhage presenting for urgent neurosurgical attention: the first involved a child with a 1-year history of slowly progressive symptoms; and the second involved a child who was asymptomatic prior to an acute change in level of

ABBREVIATIONS EVD = external ventricular drain; GCS = Glasgow Coma Scale; ICP = intracranial pressure; PA = pilocytic astrocytoma; PCR = polymerase chain reaction; VEGF = vascular endothelial growth factor.

SUBMITTED February 4, 2015. ACCEPTED October 1, 2015.

INCLUDE WHEN CITING Published online December 18, 2015; DOI: 10.3171/2015.10.PEDS1580. 
consciousness and progressive obtundation. The relevant histopathological features of each tumor pertaining to the putative pathogenesis of the hemorrhages, including the expression of vascular endothelial growth factor (VEGF), are discussed.

\section{Case Report \\ Case 1}

This 12-year-old boy presented to several physicians over the course of a year with complaints of recurrent headaches, nausea, and vomiting. These symptoms were at first attributed to a neurodevelopmental/neuropsychiatric disorder and later to a gastrointestinal source. He then developed progressive ataxia and increasing lethargy before suddenly losing consciousness at home after the onset of a severe headache. An initial noncontrast CT scan showed a midline solid and cystic cerebellar mass with recent intratumoral focal hemorrhage and obstructive hydrocephalus (Fig. 1). He was intubated, hyperventilated, and administered mannitol and 3\% hypertonic saline at a regional hospital prior to being transferred to our tertiary care center. At presentation he had a Glasgow Coma Scale (GCS) score of 3/15 with absent brainstem reflexes. Following discussion with the family, urgent placement of an external ventricular drain (EVD) was performed with drainage of $200 \mathrm{~cm}^{3}$ of cerebrospinal fluid. A postprocedural MRI study demonstrated reduced ventricular size and brainstem compression. Although his initial intracranial pressure (ICP) of $70 \mathrm{~mm} \mathrm{Hg}$ was relieved, his neurological findings remained unchanged, and he was declared clinically brain dead the following day.

Autopsy disclosed within the posterior vermis and right paramedian region a nodular PA, with an associated cyst, containing a fresh 2-cm hematoma (Figs. 2 and 3). In proximity, there was a condensed mass of hyalinized blood vessels, one of which displayed microaneurysm formation (Fig. 4). Mass effect had caused supratentorial displacement of the anterior vermis as well as compression of the medulla by the adjacent tonsils. Moderate hydrocephalus was confirmed in the cerebrum. Immunohistochemistry to detect the expression of VEGF revealed moderate immunolabeling in patchy fields of piloid cells within the cyst wall but not the lining (Fig. 5). Labeling of a few individual tumor cells in a subarachnoid deposit was similarly noted. However, the majority of the tumor

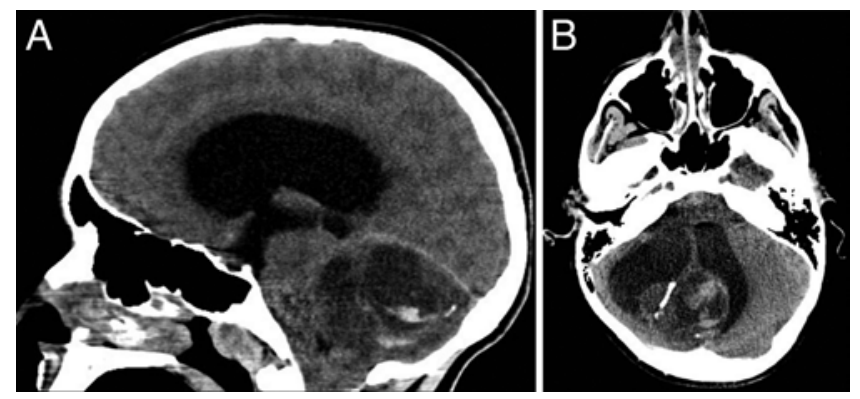

FIG. 1. Case 1. Sagittal (A) and axial (B) noncontrast CT images showing a midline mixed solid and cystic cerebellar mass with recent focal intratumoral hemorrhage and obstructive hydrocephalus.

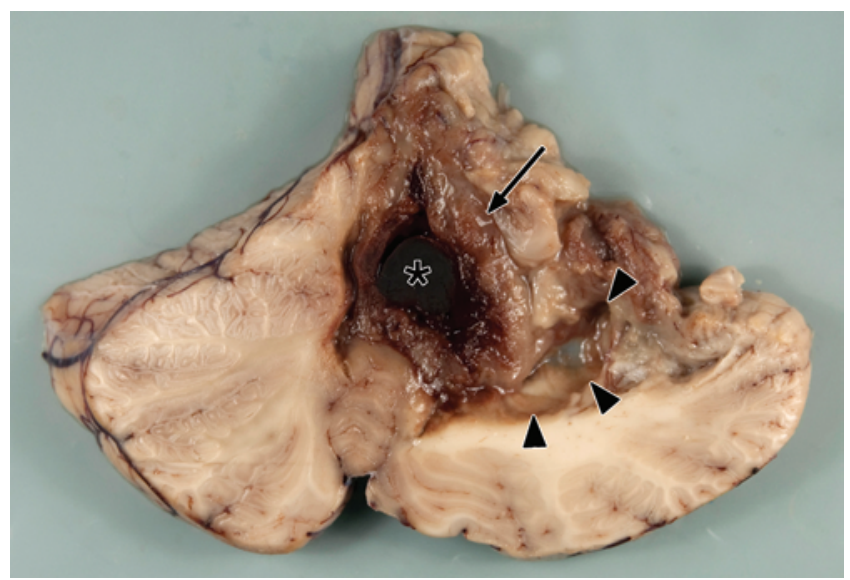

FIG. 2. Case 1. Photograph of transverse section of the cerebellum showing a $4.5 \times 2.5 \times 4-\mathrm{cm}$ mass (arrow) in the posterior vermis with a central fresh hematoma (asterisk) and bordering cyst (arrowheads) in the right hemisphere. Figure is available in color online only.

cells failed to express this marker. Immunoexpression in blood vessels was restricted to the endothelium in a cluster of telangiectatic vessels with mural hyalinization. Molecular analysis of this tumor for the presence of the most common KIAA1549:BRAF fusion variants using a custom codeset and nanoString technology (nanoString Technologies, Inc.) was undertaken. The panel allows for detection of KIAA1549 (exon 13):BRAF (exon 9), KIAA1549 (exon 15): BRAF (exon 10), KIAA1549 (exon 15):BRAF (exon 11), KIAA1549 (exon 15):BRAF (exon 9), KIAA1549 (exon 16):BRAF (exon 10del74), KIAA1549 (exon 16):BRAF (exon 11), KIAA1549 (exon 16):BRAF (exon 9), KIAA1549 (exon 18):BRAF (exon 10), and KIAA1549 (exon 19):BRAF (exon 9). After good quality RNA was confirmed, the assay was run, and none of the tested fusions was detected in the total RNA extracted from the tumor. Analysis was extended to detection of a possible $B R A F$ (V600E) mu-

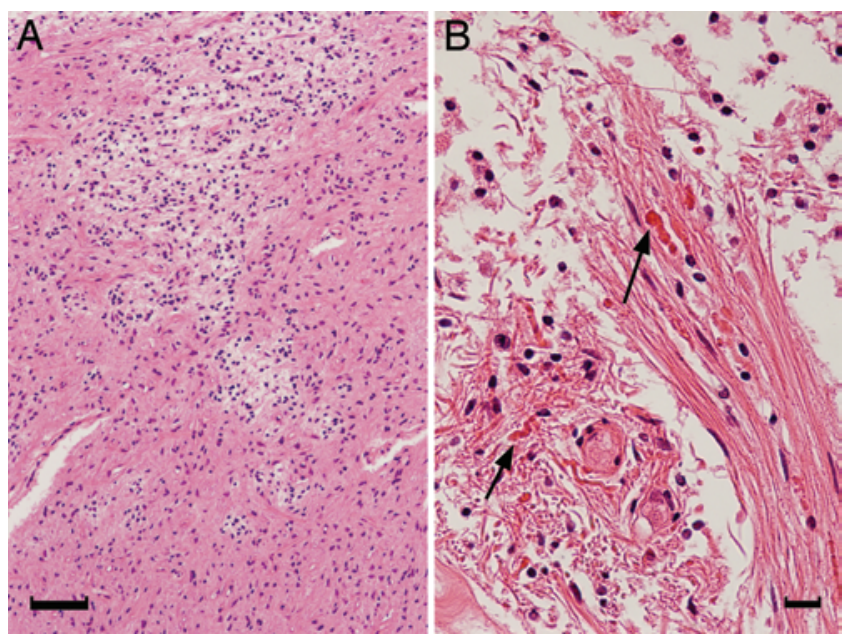

FIG. 3. Case 1. A: Photomicrograph showing pathognomonic biphasic differentiation of a PA with compact fibrillary regions merging into central microcystic fields. $H \& E$, bar $=100 \mu \mathrm{m}$. B: Photomicrograph showing bundles of neoplastic piloid astroglia in looser microcystic regions are grouped around vascular pedicles; Rosenthal fibers (arrows) are common. $\mathrm{H} \& \mathrm{E}$, bar $=20 \mu \mathrm{m}$. 


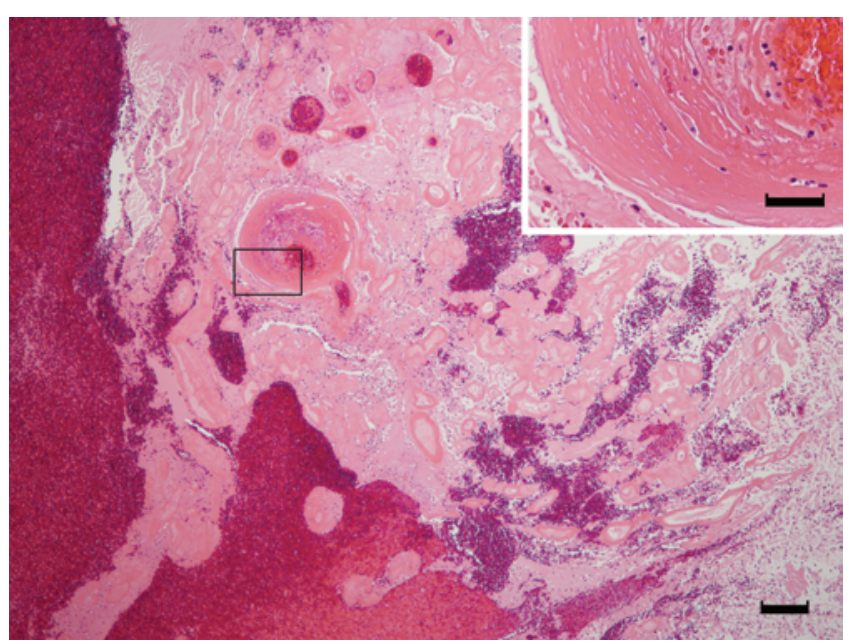

FIG. 4. Case 1. Photomicrograph showing hematoma (left) bordered by a collapsed telangiectatic array of empty sclerotic hyalinized blood vessels. A large channel shows microaneurysmal dilation and mural fibrinoid necrosis (inset), predisposing to rupture and hemorrhage. $\mathrm{H} \& \mathrm{E}$, bar = $200 \mu \mathrm{m}$, inset bar $=50 \mu \mathrm{m}$.

tation by polymerase chain reaction (PCR) amplification technique using DNA extracted from paraffin-embedded tissue. No mutation was identified.

\section{Case 2}

An otherwise healthy 5-year-old girl had a single episode of severe headache 2 weeks prior to an abrupt loss of consciousness after toileting at school. She regained consciousness but, after being taken home for observation, became increasingly obtunded over several hours before admission to hospital with a GCS score of 10/15. An initial CT scan revealed a posterior fossa mass. Mannitol was given prior to a preoperative MRI scan that disclosed a large nonenhancing cerebellar mass within the vermis and left hemisphere. Fresh intratumoral hemorrhages dotted the mass, which extended into the cisterna ambiens and compressed the brainstem, causing obstructive hydrocephalus

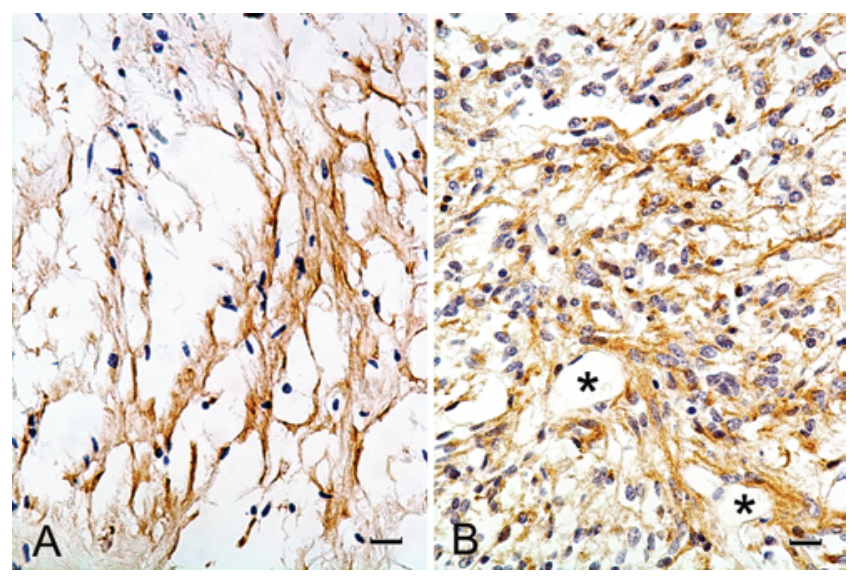

FIG. 5. VEGF immunohistochemistry. A: Case 1. Photomicrograph showing immunoexpression for VEGF in a field of piloid tumor cells from a spongiotic region of the cyst wall. B: Case 2. Photomicrograph showing VEGF immunolabeling of numerous tumor cells that highlight the walls of vascular channels (asterisks). Bar $=20 \mu \mathrm{m}$ (A and B).
(Fig. 6). A witnessed seizure occurred immediately after this scan, necessitating intubation and ventilatory support; thereafter, her pupils became bilaterally dilated and nonreactive. After insertion of an EVD, subtotal surgical decompressive resection of the tumor followed, the extent limited by the vascularity of the tumor capsule. However, in the postoperative period the patient's pupils remained nonreactive and brainstem reflexes unelicitable. CT and MRI scans showed findings consistent with diffuse ischemic/hypoxic injury involving the brainstem, deep nuclear gray matter, and cortex of the parieto-occipital regions, bilaterally. Therefore, after consultation with the family, the patient's medical management was discontinued, with death occurring shortly thereafter.

The tumor, in the surgical specimen and at autopsy, was consistent with a PA with a variant pattern of growth and containing multifocal coalescing sheets of fresh hemorrhage (Fig. 7). In keeping with a PA, it showed a biphasic differentiation consisting of mild to moderately hypercellular sheets of small bipolar spindle cells with glial fibrillary acidic protein (GFAP)- and microtubule-associated protein 2 (MAP-2)-immunoreactive somas and processes arrayed in loosely reticulated spongiotic sheets that condensed into focally compacted fibrillary fields; scattered calcospherites were common. The proportion of these 2 patterns varied from region to region. Along the limited margin of invasion in the left middle cerebellar peduncle, fibrillary differentiation predominated with an intermixture of oligodendroglia-like cells. However, within the infiltrated subarachnoid space of the left cerebellopontine angle, the growth pattern was spongiotic. This leptomeningeal spread continued into the interpedunclar cistern and the hippocampal fissures bilaterally. Nonetheless, Rosenthal fibers and eosinophilic granular bodies were absent; and the tumor did not show a predominant angiocentric pattern. No $I D H-1$ or $I D H-2$ mutations were found in the surgical specimen by PCR amplification analysis, and immunoexpression for p53 was less than $10 \%$. As with the first case, no KIAA1549:BRAF fusion or BRAF (V600E) mutation was detected. Immunohistochemistry in the surgically resected tissue revealed moderate to marked cytoplasmic expression for VEGF in the majority of the
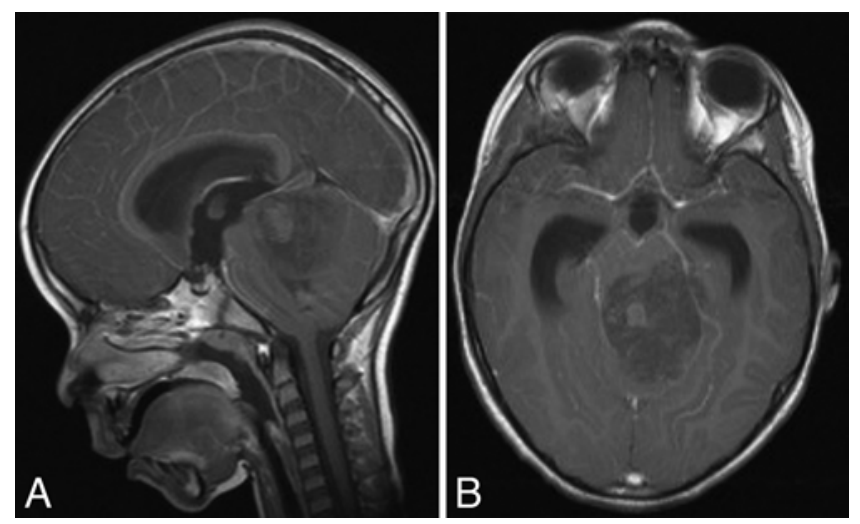

FIG. 6. Case 2. Sagittal (A) and axial (B) T1-weighted MR images showing a nonenhancing cerebellar mass with recent patchy intratumoral hemorrhage, associated brainstem compression, and obstructive hydrocephalus. 


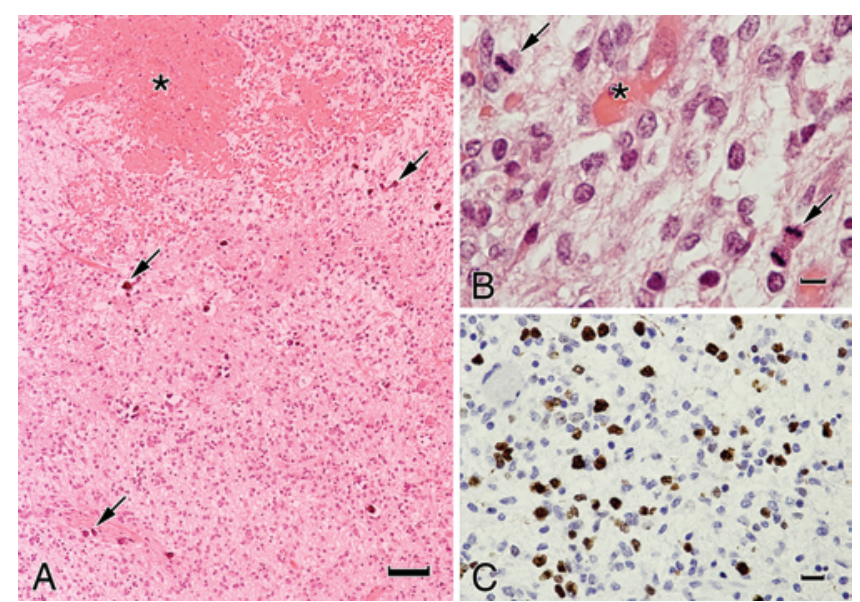

FIG. 7. Case 2. Surgical biopsy specimen. A: Photomicrograph showing sheets of fresh hemorrhage (asterisk) bordering a compacted fibrillary region that blends into a loosely spongiotic field, center. Several calcospherites (arrows) are present. H \& E, bar $=100 \mu \mathrm{m}$. B: Highmagnification photomicrograph showing bipolar tumor cells with mild nuclear pleomorphism, mitotic figures (arrows), and congested capillary (asterisk). H \& E, bar = $10 \mu \mathrm{m}$. C: MIB-1 proliferation analysis showing approximately $20 \%$ of tumor cells with labeled nuclei (black). MIB-1 immunohistochemistry, bar $=20 \mu \mathrm{m}$.

neoplastic cells (Fig. 5). However, in the autopsy tissue, immunolabeling was weaker and limited to patchy groups of cells. Mitotic figures were common (maximum mitotic index 6 to 8 mitoses per 10 high-power microscopic fields) and the MIB-1 labeling index ranged from $10 \%$ to $20 \%$ (Fig. 6). Correlating with the postoperative imaging studies were the findings of hemorrhagic infarcts in the pons and medulla, and global hypoxic necrosis of the cerebral cortices and subcortical gray matter structures.

\section{Discussion}

The cerebellar tumors in these 2 children illustrate the diverse features of PAs that can be associated with spontaneous hemorrhage. The tumor in the first case displays the conventional gross and microscopic features of a PA: cyst with mural nodule; biphasic pattern of growth; associated findings of Rosenthal fibers and eosinophilic granular bodies; and the regressive change of vascular hyalinization. In the second case, the diagnosis of PA is based on the presence of a mild to moderately hypercellular biphasic pattern with a solid fibrillary margin of infiltration, replete with interspersed oligodendroglia-like cells, in the left middle cerebellar peduncle. Although the predominance of spongiotic differentiation in some fields, in association with the absence of Rosenthal fibers and eosinophilic granular bodies, is suggestive of pilomyxoid astrocytoma in the differential diagnosis, this possibility is less tenable because of the lack of the pathognomonic angiocentric pattern. Moreover, Rosenthal fibers and eosinophilic granular bodies are not consistent findings in all PAs. Diffuse astrocytomas can arise in the cerebellum but, similar to their supratentorial counterparts, are predominately fibrillary in growth with broad borders of parenchymal infiltration, unlike that noted in this case, and are more common in adolescents and adults. Further tending to exclude the possibility of a diffuse astrocytoma is the low expression of p53 and absence of detectable mutations in $I D H-1$ and $I D H-2$. The interpretive significance of mitotic figures in PAs, as in this tumor, has been moot. Although there has been discussion in the literature on the correlation between clinical outcome and MIB-1 index and mitotic indices, there has been no unequivocal consensus of the impact on progression-free and overall survival. ${ }^{22}$ Nor has the WHO Classification of Tumors of the Central Nervous System as yet provided clear guidelines for designating tumors with high mitotic/MIB-1 indices as "atypical" or "anaplastic." tive classification of the tumor in this second case, other than pilocytic astrocytoma (PA), is not resolvable.

The genetic abnormality most commonly associated with PAs is KIAA1549:BRAF fusion rearrangement at $7 \mathrm{q} 34$, notably in those tumors that arise in the cerebellum. However, none of the most common KIAA 1549:BRAF fusion events was detected in the tumors of either of these patients. This finding is in keeping with previous studies suggesting a more aggressive clinical course in those PAs lacking the KIAA1549:BRAF fusion. ${ }^{2,10}$ It is possible that a rarer gene fusion event (i.e., FAM131B:BRAF or SRGAP3:RAF1) may have been operant. Even though the $B R A F$ point mutation resulting in the V600E amino acid substitution has further been excluded in these 2 tumors, mutations of other genes (e.g., PTEN, p16, etc.) involving different signaling pathways may have been involved but could not be assessed due to limitations in the amount of tissue available. For these reasons, the inability to detect a KIAA1549:BRAF fusion rearrangement or the BRAF (V600E) mutation in either tumor does not obviate the diagnosis of PA.

Once considered an infrequent occurrence, ${ }^{8,21,23}$ spontaneous hemorrhage in PAs has become more recognized $^{1,9,11,12,15,19,24}$ and can present as an acute neurosurgical emergency. In a retrospective review of 138 cases of PAs, White et al. ${ }^{24}$ reported that $8 \%$ of their patients had a concurrent hemorrhage at presentation as assessed by CT and MRI and confirmed by pathological examination of the resected tumor. These bleeds were often heralded by onset of new symptoms but tended to be small and without preference for the site of the tumor, although none occurred in the cerebellar PA cases (proportion not stated in this study). In concordance, a review by Shibahara et al. ${ }^{19}$ of 35 cases of PA or pilomyxoid astrocytoma found the presence of an intratumoral hemorrhage on imaging studies in $11.4 \%$. Two of the 4 hemorrhages in this study occurred in the cerebellum, the site of 10 tumors in total. Analysis of the clinical presentation of the 2 hemorrhagic cerebellar PAs in the review of Shibahara et al. in combination with 7 other known reported cases of cerebellar PAs presenting with hemorrhage ${ }^{8,9,11,12,15,21,23}$ reveals that 3 of 9 patients ${ }^{11,15,19}$ had complaints over a 10-day to 4 -week period prior to their hemorrhagic presentation (Table 1). These complaints were referable to cerebellar dysfunction, often accompanied by headache and features suggesting increased ICP prior to the abrupt deterioration of the patients' condition. This scenario mirrors that of our first case, which in hindsight demonstrates how subtle development of complaints over a prolonged period can lead to 
TABLE 1. Published cases describing hemorrhagic presentations of cerebellar PA

\begin{tabular}{|c|c|c|c|c|c|c|}
\hline PA Cases & Age & Sex & Cerebellar Location & Hemorrhage Location & Duration of Preceding CNS Sx & Survival \\
\hline \multicolumn{7}{|c|}{ w/ presentations prior to acute event } \\
\hline Case 1, present publication & 12 yrs & M & Vermis & IT & $1 \mathrm{yr}$ & No \\
\hline Kumar et al., 2010 & 16 yrs & $\mathrm{F}$ & Rt hemisphere & IT & $10-15$ days & Yes \\
\hline Mesiwala et al., 2001 & 13 yrs & M & Lt hemisphere & IT & 10 days & Yes \\
\hline Shibahara et al., 2009 & 8 yrs & M & Lt hemisphere & IT & 4 wks & Yes \\
\hline \multicolumn{7}{|l|}{ w/ no prior presentations } \\
\hline Case 2, present publication & $5 \mathrm{yrs}$ & $\mathrm{F}$ & Vermis/lt hemisphere & IT, SAH & 1 remote severe $\mathrm{HA}$ & No \\
\hline Fogelson et al., 1980 & $9 \mathrm{yrs}$ & $\mathrm{F}$ & Vermis & IT & NA & Yes \\
\hline Frassanito et al., 2009 & $7 \mathrm{yrs}$ & $\mathrm{F}$ & Quadrigeminal plate/vermis & IT, SAH & NA & Yes \\
\hline Lee et al., 2009 & $15 \mathrm{mos}$ & M & Lt hemisphere & IT, SAH, SDH & NA & Yes \\
\hline Shibahara et al., 2009 & $22 \mathrm{yrs}$ & M & Rt tonsil & IT & NA & Yes \\
\hline Specht et al., 1986 & 8 yrs & M & Vermis/rt hemisphere & IT, SAH, IVH & 2 prior HAs, 2 prior falls & No \\
\hline Vincent et al., 1980 & 14 yrs & $\mathrm{F}$ & Vermis/rt hemisphere & IT & NA & Yes \\
\hline
\end{tabular}

HA = headache; IT = intratumoral; IVH = intraventricular hemorrhage; NA = not available; SAH = subarachnoid hemorrhage; SDH = subdural hematoma; Sx = symptoms.

misattribution to alternative etiologies in the differential diagnosis. The other 6 patients ${ }^{8,9,12,19,21,23}$ had acute presentations with symptoms and/or signs of elevated ICP. In 3 of these patients there were associated cerebellar signs, and a facial palsy in one. Of note, similar to our second case, one of these patients ${ }^{21}$ had 2 episodes of vomiting and 2 falls in the preceding 2 weeks prior to admission. This case was the only reported fatality and, as with our second case, warns that seemingly minor transient signs can deceptively precede a catastrophic acute ictus.

As with hemorrhages occurring in high-grade gliomas, those reported in PAs have been repeatedly ascribed to the interaction of a variety of factors that predispose, initiate, and propagate bleeding. Often cited factors include rate of tumor growth, tumor invasion of blood vessels, necrosis of blood vessels and/or tumor, blood coagulability and local fibrinolysis, intrinsic structural features of the tumor vasculature, and the presence of vascular proliferation. However, the role of these individual factors and the mechanism of their interactions have been speculative to date. An exception is a report by Aichholzer et al. ${ }^{1}$ of the rupture of a radiation-induced aneurysm of the anterior communicating artery into an optic chiasm PA. A morphological study by Liwnicz et al., ${ }^{14}$ nonetheless, did suggest a relationship between the structural organization and mechanical support of the capillary bed, glioma type, and propensity for hemorrhage. In their analysis, they found a statistically significant association between the presence of a retiform capillary pattern and spontaneous bleeding in glioblastomas and low-grade astrocytomas but not in oligodendrogliomas. Hemorrhages in oligodendrogliomas, however, did have a statistically significant correlation with deposits of perivascular calcification (calcospherites). In their review, White et al. ${ }^{24}$ noted a similar association, with calcospherites being present in $27 \%$ of the PAs with hemorrhage, a relationship that suggests a predisposing factor. Other associated vascular changes included the concurrence of thin-walled ectatic blood vessels, degenerative mural hyalinization of vessels, and "glomeruloid" endothelial hyperplasia.
Further insight into the pathogenesis of these vascular alterations and the risk for bleeding can be inferred from research in glioma-induced angiogenesis, ${ }^{5,7}$ specifically that in PAs. A key promoter in angiogenesis is the upregulated expression of VEGF in response to regional tissue hypoxia as mediated by hypoxia-inducible factor-1. In a comparative immunohistochemical morphometric analysis of the vasculature in PAs versus that in glioblastomas, Sie et al. ${ }^{20}$ noted an overlapping increased expression of VEGF in both types of glioma. However, in the PA group the level of this expression inversely correlated with the density of the microvasculature, implicating the role of local tissue hypoxia as the agent for upregulation. By way of compensation, the microvessels in the PA group were wider (i.e., ectatic). Even though these vessels were also more mature, in the subset of tumors arising in the cerebellum the integrity of the vasculature was as unstable as that in glioblastomas, suggesting these blood vessels to be more prone to breakdown. This study corroborates an earlier report by Leung et al. ${ }^{13}$ of elevated VEGF expression in PAs, which by in situ hybridization was localized to tumor cells in the microcystic/spongiotic regions, cyst wall, and border zones of degenerative vascular hyalinization, parts of the glioma more predisposed to a hypoxic milieu. In correspondence, upregulation of the receptors for VEGF was observed in the endothelia of proliferating capillaries in these regions. Because VEGF is a potent agent for increasing vascular permeability, as well as being a mediator for the recruitment and proliferation of endothelial cells, these authors propose that the degenerative changes of cyst formation and vascular hyalinization are referable to this hyperpermeablility, whereby the integrity of the vessel wall would be disrupted by the transudation of plasma proteins and coagulation factors in conjunction with activation of factors involved in the remodeling of the extracellular matrix. In this setting, it is hypothesized that regional tissue hypoxia becomes chronic and perpetuates a self-sustaining feedback loop for persistent VEGF secretion. That augmented VEGF expression may be relevant to the risk of bleeding in PAs is given further cre- 
dence by the demonstration of the role of VEGF in inducing intratumoral hemorrhage in an in vivo experimental glioblastoma model in mice. ${ }^{4}$ In this study, the occurrence and size of spontaneous hemorrhage had a dose-dependent association to the amount of VEGF expression, provided that expression was sustained at a constant level over time. This hemorrhagic phenomenon appeared to be related to the intensity of the induced neoangiogenesis.

The observation of VEGF expression in the tumors of these 2 children, therefore, has relevance toward a predisposition to spontaneous hemorrhage. In the first case, similar to that reported by Leung et al., ${ }^{13}$ VEGF is observed in patchy groups of tumor cells comprising the cyst wall and, less commonly, in sporadic cells in a more compacted subarachnoid deposit. Expression is further noted in the endothelium of hyalinized telangiectatic blood vessels. In the second case, there is diffuse expression of VEGF by the neoplastic cells. As illustrated by this case, however, postmortem autolysis can modify the detection of VEGF in that the immunoreactivity in the autopsy tissue from this patient was less consistent in amount and distribution compared with that obtained during resective surgery. Hence, expression in the PA of the first patient may have been more intense and widespread had it not been for autolysis. Factors contributing to the amount of autolysis would be the prolonged periods both patients were sustained on assisted ventilation after ictus, associated compromised perfusion of posterior fossa structures, and lengthy postmortem intervals. The crux, nonetheless, is that both tumors expressed VEGF, which is not reported in low-grade gliomas other than PAs. Whether PAs with hemorrhage have higher levels of VEGF expression compared with tumors without hemorrhage is unknown. To resolve this question, a comparative study with a more analytical methodology other than semiquantitative immunohistochemistry would be required.

In the context of the preceding discussion, the following suppositions can be proposed as the likely causes of hemorrhage in our 2 cases. In the first case, it can be presumed that in the coalescing fields of degenerative blood vessels, altered flow dynamics were ongoing as a consequence of the progressive obliterative sclerosis of the hyalinized blood vessels, further stressing the vasculature. Some of these vessels, in response, underwent fibrinoid necrosis and microaneurysm formation, as illustrated. Thus, rupture of such a microaneurysm would be the most plausible explanation for the patient's intratumoral hemorrhage and the abrupt deterioration of his condition. In the second case, although causation of hemorrhage is more obscure, factors for consideration include large size and rapid growth of the tumor, predominance of the background myxoid matrix, and the thin-walled tumor vasculature. These factors could predispose to regional hypoxia with the induced expression of VEGF that undermined the stability of the blood vessel walls, as suggestively inferred by the presence of calcospherites. In this setting, changes in vascular flow dynamics and hydrostatic pressure, as could occur with a Valsalva maneuver during toileting, possibly caused leakage to initiate microhemorrhages that coalesced into larger hemorrhages. This mechanism plausibly explains the slow progression of the patient's obtun- dation and correlates with the punctate pattern of bleeding observed in the imaging studies. Of interest in the tumors of both patients is the absence of an identifiable $B R A F$ fusion rearrangement or mutation. The significance of this finding to the occurrence of spontaneous hemorrhage, however, remains undefined.

\section{Conclusions}

As documented by these 2 case examples and our review of the literature, spontaneous bleeding in cerebellar PAs can manifest in 2 patterns of presentation. One scenario is the progressive expression of symptoms and signs of cerebellar dysfunction, and on occasion raised ICP, over weeks to months, masquerading as other disorders. The other, more common, scenario is a precipitant presentation with features of life-endangering increased ICP. In some instances, the source of hemorrhage can be ascribed to specific pathological changes in the tumor vasculature; in other instances, based on current research on gliomainduced angiogenesis, it can only be inferred that various angiogenic factors are operant to destabilize the vasculature to promote bleeding. As mentioned by White et al., ${ }^{24}$ these hemorrhages probably occur more frequently than assumed, are usually small, but can quickly expand into large bleeds with rapid clinical progression. As with the previously reported cases, our 2 cases are cautionary illustrations of the precarious situation that hemorrhagic presentations of tumors pose, demonstrating that sudden fluctuations in an already tense posterior fossa can abruptly devolve into devastating outcomes.

\section{References}

1. Aichholzer M, Gruber A, Haberler C, Bertalanffy A, Slave I, Czech T: Intracranial hemorrhage from an aneurysm encased in a pilocytic astrocytoma-case report and review of the literature. Childs Nerv Syst 17:173-178, 2001

2. Becker AP, Scapulatempo-Neto C, Carloni AC, Paulino A, Sheren J, Aisner DL, et al: KIAA1549:BRAF gene fusion and FGFR1 hotspot mutations are prognostic factors in pilocytic astrocytomas. J Neuropathol Exp Neurol 74:743-754, 2015

3. Burkhard C, Di Patre PL, Schüler D, Schüler G, Yaşargil MG, Yonekawa Y, et al: A population-based study of the incidence and survival rates in patients with pilocytic astrocytoma. J Neurosurg 98:1170-1174, 2003

4. Cheng SY, Nagane M, Huang HS, Cavenee WK: Intracerebral tumor-associated hemorrhage caused by overexpression of the vascular endothelial growth factor isoforms $\mathrm{VEGF}_{121}$ and $\mathrm{VEGF}_{165}$ but not $\mathrm{VEGF}_{189}$. Proc Natl Acad Sci U S A 94:12081-12087, 1997

5. Dvorak HF: Angiogenesis: update 2005. J Thromb Haemost 3:1835-1842, 2005

6. Fernandez C, Figarella-Branger D, Girard N, Bouvier-Labit C, Gouvernet J, Paz Paredes A, et al: Pilocytic astrocytomas in children: prognostic factors-a retrospective study of 80 cases. Neurosurgery 53:544-555, 2003

7. Fischer I, Gagner JP, Law M, Newcomb EW, Zagzag D: Angiogenesis in gliomas: biology and molecular pathophysiology. Brain Pathol 15:297-310, 2005

8. Fogelson MH, Oppenheim RE, McLaurin RL: Childhood cerebellar astrocytoma presenting with hemorrhage. Neurology 30:669-670, 1980

9. Frassanito P, Massimi L, Caldarelli M, Di Rocco C: Cerebellar mutism after spontaneous intratumoral bleeding involving 
the upper cerebellar vermis: a contribution to the physiopathogenic interpretation. Childs Nerv Syst 25:7-11, 2009

10. Hawkins C, Walker E, Mohamed N, Zhang C, Jacob K, Shirinian M, et al: BRAF-KIAA1549 fusion predicts better clinical outcome in pediatric low-grade astrocytoma. Clin Cancer Res 17:4790-4798, 2011

11. Kumar A, Deopujari CE, Biyani N, Mhatre MV: Pediatric cerebellar pilocytic astrocytoma presenting with hemorrhage. Neurol India 58:972-974, 2010

12. Lee CS, Huh JS, Sim KB, Kim YW: Cerebellar pilocytic astrocytoma presenting with intratumor bleeding, subarachnoid hemorrhage, and subdural hematoma. Childs Nerv Syst 25:125-128, 2009

13. Leung SY, Chan ASY, Wong MP, Yuen ST, Cheung N, Chung LP: Expression of vascular endothelial growth factor and its receptors in pilocytic astrocytoma. Am J Surg Pathol 21:941-950, 1997

14. Liwnicz BH, Wu SZ, Tew JM Jr: The relationship between the capillary structure and hemorrhage in gliomas. J Neurosurg 66:536-541, 1987

15. Mesiwala AH, Avellino AM, Roberts TS, Ellenbogen RG: Spontaneous cerebellar hemorrhage due to a juvenile pilocytic astrocytoma: case report and review of the literature. Pediatr Neurosurg 34:235-238, 2001

16. Ostrom QT, Gittleman H, Farah P, Ondracek A, Chen Y, Wolinsky Y, et al: CBTRUS statistical report: Primary brain and central nervous system tumors diagnosed in the United States in 2006-2010. Neuro Oncol 15 (Suppl 2):ii1-ii56, 2013

17. Rodriguez FJ, Scheithauer BW, Burger PC, Jenkins S, Giannini C: Anaplasia in pilocytic astrocytoma predicts aggressive behavior. Am J Surg Pathol 34:147-160, 2010

18. Scheithauer BW, Hawkins C, Tihan T, VandenBerg SR, Burger PC: Pilocytic astrocytoma, in Louis DN, Ohagaki H, Wiestler OD et al (eds): WHO Classification of Tumors of the Central Nervous System. Lyon, France: IARC, pp 14-20, 2007

19. Shibahara I, Kanamori M, Kumabe T, Endo H, Sonoda Y, Ogawa Y, et al: Hemorrhagic onset of pilocytic astrocytoma and pilomyxoid astrocytoma. Brain Tumor Pathol 26:1-5, 2009

20. Sie M, de Bont ESJM, Scherpen FJG, Hoving EW, den Dun- nen WFA: Tumour vasculature and angiogenic profile of paediatric pilocytic astrocytoma; is it much different from glioblastoma? Neuropathol Appl Neurobiol 36:636-647, 2010

21. Specht CS, Pinto-Lord C, Smith TW, DeGirolami U, Suran E, Marshall PC, et al: Spontaneous hemorrhage in a mixed glioma of the cerebellum: case report. Neurosurgery 19:278-281, 1986

22. Tibbetts KM, Emnett RJ, Gao F, Perry A, Gutmann DH, Leonard JR: Histopathologic predictors of pilocytic astrocytoma event-free survival. Acta Neuropathol 117:657-665, 2009

23. Vincent FM, Bartone JR, Jones MZ: Cerebellar astrocytoma presenting as a cerebellar hemorrhage in a child. Neurology 30:91-93, 1980

24. White JB, Piepgras DG, Scheithauer BW, Parisi JE: Rate of spontaneous hemorrhage in histologically proven cases of pilocytic astrocytoma. J Neurosurg 108:223-226, 2008

\section{Disclosures}

The authors report no conflict of interest concerning the materials or methods used in this study or the findings specified in this paper.

\section{Author Contributions}

Conception and design: Wilson, Alshaya, Pugh. Acquisition of data: Hawkins. Analysis and interpretation of data: Hawkins. Drafting the article: Wilson, Johnson, Atkins, Alshaya, Pugh. Critically revising the article: Wilson, Johnson, Hawkins, Pugh. Reviewed submitted version of manuscript: Wilson, Johnson, Hawkins, Atkins, Pugh. Approved the final version of the manuscript on behalf of all authors: Wilson. Administrative/technical/ material support: Wilson, Johnson, Hawkins, Pugh.

\section{Correspondence}

Mitchell P. Wilson, Department of Radiology and Diagnostic Imaging, University of Alberta, 2C2.41 WMC, 8440-112 St., Edmonton, AB T6G2B7, Canada. email: mitch.wilson@ualberta. ca. 